\title{
Review: isolated systolic hypertension increases mortality and morbidity in elderly people and should be treated
}

\author{
Staessen JA, Gasowski J, Wang JG, et al. Risks of untreated and treated isolated systolic hypertension in the elderly: \\ meta-analysis of outcome trials. Lancet 2000 Mar 11;355:865-72.
}

QUESTIONS: In elderly people, what are the risks associated with isolated systolic hypertension ( $\geq 160 \mathrm{~mm} \mathrm{Hg}$ with diastolic blood pressure $[\mathrm{BP}]<95 \mathrm{~mm} \mathrm{Hg}$ ), and what is the magnitude of the benefit associated with treatment?

\section{Data sources}

Studies were identified from 10 published overviews and 2 reports from trialist collaborations.

\section{Study selection}

Controlled trials were selected if elderly people with isolated systolic hypertension were enrolled. Trials were excluded if all the study participants had comorbid conditions, such as stroke; if specialised care was compared with routine antihypertensive agents; and if the study was a preliminary evaluation for a larger study.

\section{Data extraction}

Data were extracted on study quality, patient characteristics, antihypertensive treatment, duration and length of follow up, and outcomes (all cause and cardiovascular mortality, cardiovascular complications, all strokes, and all coronary events). Strokes did not include transient ischaemic attacks. Cardiovascular complications included coronary artery disease (myocardial infarction and sudden death), stroke, and vascular disorders.

\section{Main results}

8 trials (15 693 patients) were included. Mean age range was 62 to 76 years, median follow up was 3.8 years, prevalence of smoking at baseline was $16 \%$, and $31 \%$ of participants had $\geq 1$ cardiovascular complication at baseline. All cause mortality was positively associated with systolic BP $(\mathrm{p}<0.001)$ and negatively associated with diastolic BP $(p=0.05)$. With any given increase in systolic BP, a lower diastolic BP increased the risk for death. For each $10 \mathrm{~mm}$ increase in systolic BP, an increase was shown in all cause mortality (hazard ratio [HR] 1.26, 95\% CI 1.13 to 1.40 ), cardiovascular death (HR 1.22, CI 1.06 to 1.40), cardiovascular events (HR 1.15 , CI 1.04 to 1.28 ), and stroke (HR 1.22, CI 1.04 to 1.40 ) but not coronary events (HR 1.07, CI 0.91 to 1.26 ). For each $5 \mathrm{~mm} \mathrm{Hg}$ increase in diastolic $\mathrm{BP}$, a decrease in all cause mortality was found (HR 0.95, CI 0.89 to 1.00 ).

With treatment, the mean reduction was 10.4 (range 6.9 to 18.2 ) in systolic BP and 4.1 (range 2.3 to 8.3 ) $\mathrm{mm}$ $\mathrm{Hg}$ in diastolic BP. An improvement in all outcomes was found when results for all trials were pooled (table). Summary analysis showed that all outcomes improved with treatment even across subgroups based on sex, age, systolic BP, pulse pressure, previous cardiovascular complications, and smoking status.

\section{Conclusions}

Isolated systolic hypertension and wide pulse pressure in elderly people increase the risk for mortality and morbidity. Treatment reduces this risk.
Treatment of isolated systolic hypertension in elderly people*

\begin{tabular}{lll}
$\begin{array}{l}\text { Outcomes at mean } \\
3.8 \text { years }\end{array}$ & $\begin{array}{l}\text { Odds ratio } \\
\text { reduction } \\
\text { (95\% Cl) }\end{array}$ & NNT (Cl) \\
\hline All cause mortality & $13 \%(2$ to 22$)$ & $59(55$ to 64$)$ \\
\hline Cardiovascular mortality & $18 \%(4$ to 29$)$ & $79(72$ to 89$)$ \\
\hline All cardiovascular events & $26 \%(17$ to 34$)$ & $26(25$ to 27$)$ \\
\hline All stroke events & $30 \%(18$ to 41$)$ & $48(45$ to 51$)$ \\
\hline All coronary events & $23 \%(10$ to 34$)$ & $64(59$ to 70$)$ \\
\hline
\end{tabular}

*Abbreviations defined in glossary. 1 trial was not a randomised controlled trial; re-analysis without this trial did not alter outcomes significantly.

\section{COMMENTARY}

Clinicians, be comforted! This sophisticated meta-analysis by Staessen et al confirms what we already know: systolic BP better predicts cardiovascular events than does diastolic BP, and absolute 5 year benefits of antihypertensive drug treatment are greater among people with higher baseline risks, such as men, older adults, and people with known cardiovascular disease. It also confirms several observational studies that show that wider pulse pressure-a measure dependent on ventricular ejection, timing of blood pressure waves, and arterial stiffness-is associated with higher baseline risks.

Should we now routinely use pulse pressure rather than systolic and diastolic BP measurements alone? Not yet. Firstly, for those who rely on risk assessment to determine when benefits of treatment are likely to outweigh harms, we need changes in current risk assessment tools to incorporate pulse pressure. Secondly, antihypertensive agents have multiple and heterogeneous effects, and the pathophysiology of hypertension is complex; all agents are not equivalent despite equivalent BP reduction. ${ }^{1}$ We do not yet know whether differences in drug benefits are caused by differences in reducing pulse pressure.

Active research to improve evidence-based care of patients with hypertension is alive and well. The results of these studies are still subject to change. What will the bottom line be? Clinicians, stand by!

Ronald T Ackermann, MD Cynthia D Mulrow, MD Audie L Murphy Memorial Veterans Hospital San Antonio, Texas, USA

1 ALLHAT Collaborative Research Group. JAMA 2000;283:1967-75 